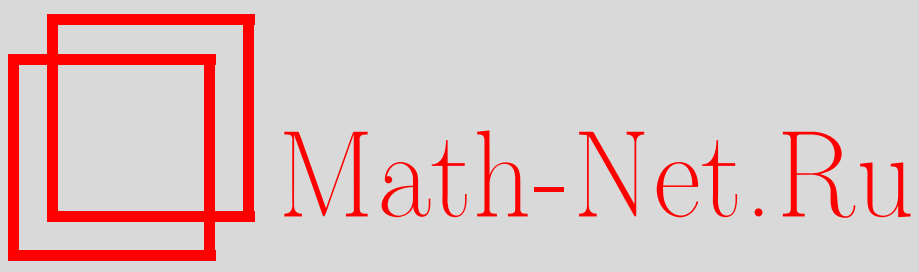

В. И. Богачев, О проблеме малых шаров для эквивалентных гауссовских мер, Maтем. сб., 1998, том 189, номер 5, 47-68

DOI: https://doi.org/10.4213/sm319

Использование Общероссийского математического портала Math-Net.Ru подразумевает, что вы прочитали и согласны с пользовательским соглашением

http: //www. mathnet.ru/rus/agreement

Параметры загрузки:

IP: 52.23 .180 .231

26 апреля 2023 г., 16:32:29 
УДК 512.55

\section{В.И. Богачев}

\section{О проблеме малых шаров для Эквивалентных гауссовских мер}

Пусть $\mu$ - центрированная гауссовская мера на линейном пространстве $X$ с пространством Камерона-Мартина $H, q-\mu$-измеримая полунорма, а $Q$ - $\mu$-измеримый многочлен второго порядка. В работе показано, что для существования предела $\lim _{\varepsilon \rightarrow 0} \mathrm{E}(\exp Q \mid q \leqslant \varepsilon)$, где $\mathrm{E}$ - математическое ожидание относительно $\mu$, достаточно, чтобы вторая производная $D_{H}^{2} Q$ функции $Q$ была ядерным оператором в $H$. Это условие является и необходимьм для существования указанного предела для всех полунорм $q$. Обсуждаемая задача может быть переформулирована следующим образом: изучить $\lim _{\varepsilon \rightarrow 0} \nu(q \leqslant \varepsilon) / \mu(q \leqslant \varepsilon)$ для гауссовских мер $\nu$, эквивалентных $\mu$.

Библиография: 23 названия.

В настоящей работе исследуется задача о существовании предела

$$
\lim _{\varepsilon \rightarrow 0} \frac{\nu(x: q(x) \leqslant \varepsilon)}{\mu(x: q(x) \leqslant \varepsilon)},
$$

где $\mu$ - центрированная гауссовская мера на локально выпуклом пространстве $X$, $\nu$ - гауссовская мера, эквивалентная $\mu$, а $q$ - произвольная $\mu$-измеримая норма (или полунорма) на $X$. Плотность Радона-Никодима меры $\nu$ относительно меры $\mu$ имеет вид $\exp Q$, где $Q-\mu$-измеримый многочлен второй степени. Основной результат работы состоит в следуюшем. Положительньй и конечный предел в (0.1) сушествует для всякой $\mu$-измеримой нормы $q$ на $X$ в точности тогда, когда вторая производная $Q$ вдоль пространства Камерона-Мартина $H=H(\mu)$, обозначаемая через $D_{H}^{2} Q$, является ядерньм оператором на $H$ (вообще говоря, $D_{H}^{2} Q$ - оператор Гильберта-Шмидта). Если $D_{H}^{2} Q=A_{1}-A_{2}$, где $A_{1}$ - неотрицательный оператор Гильберта-Шмидта на $H$ с бесконечным следом, а $A_{2}$ - ядерньй оператор на $H$, то предел в (0.1) равен нулю, а если $D_{H}^{2} Q=A_{2}-A_{1}$, то этот предел равен бесконечности. В остальных случаях всегда можно так подобрать норму $q$, что отношение в (0.1) не будет иметь определенного предела при стремлении $\varepsilon$ к нулю. Данная задача обсуждалась многими авторами (см. [1]-[6] и имеющиеся там ссылки). Наиболее общий известный ранее результат был получен в [5], где было установлено существование предела в (0.1) при условии, равносильном ядерности $D_{H}^{2} Q$ (см. пояснения ниже), и при дополнительном условии ограниченности множеств $\{q \leqslant \varepsilon\}$. В случае, когда меры $\mu$ и $\nu$ отличаются лишш средним, обсуждаемая проблема может быть сформулирована как задача о существовании функций Онзагера-Маклупа; ее положительное решение дано в [7], [8]. Отметим, что для

Работа вьполнена при поддержке Российского фонда фундаментальных исследований (гранты № № 96-15-96865, 97-01-00932), германского гранта 436 RUS 113/343/0(R), а также грантов INTAS 94-0378, INTAS-RFBR 95-0099. 
многих конкретных мер $\mu$ и полунорм $q$ известны довольно точные двусторонние оценки величин $\mu(q \leqslant \varepsilon)$ (см. [2], [3], [5], [9]-[12] и имеющиеся там ссылки). В этих случаях существование предела в $(0.1)$ позволяет оценить и $\nu(q \leqslant \varepsilon)$.

Наконец, стоит заметить, что основной результат работы можно эквивалентным образом сформулировать в терминах последовательностей гауссовских случайных величин. А именно, пусть $\left\{\xi_{n}\right\}$ и $\left\{\eta_{n}\right\}$ - совместно гауссовские центрированные случайные величины, причем $\xi_{n}$ - стандартные независимые гауссовские случайные величины. Предположим, что случайная величина $q=\sup _{n}\left|\eta_{n}\right|$ конечна почти наверное и $\{q<\varepsilon\}$ имеет положительную вероятность для всякого $\varepsilon>0$. Пусть $c, c_{n}, \alpha_{n}$ - вешественные числа, причем

$$
\sum_{n=1}^{\infty} c_{n}^{2}<\infty, \quad \sum_{n=1}^{\infty} \alpha_{n}^{2}<\infty, \quad \alpha_{n}<\frac{1}{2} .
$$

Пусть

$$
Q=c+\sum_{n=1}^{\infty} c_{n} \xi_{n}+\sum_{n=1}^{\infty} \alpha_{n}\left(\xi_{n}^{2}-1\right)
$$

Отметим, что соответствующие ряды сходятся почти наверное и в среднем квадратическом. Положим $J_{\varepsilon}=\mathrm{E}(\exp Q \mid q \leqslant \varepsilon)$. Тогда конечньй и положительный предел $\lim _{\varepsilon \rightarrow 0} J_{\varepsilon}$ существует при любом выборе $q$ в точности тогда, когда $\sum_{n=1}^{\infty}\left|\alpha_{n}\right|<\infty$. Если $\alpha_{n}^{+}$и $\alpha_{n}^{-}$есть соответственно положительные и отрицательные элементы последовательности $\left\{\alpha_{n}\right\}$, то сходимость одного из рядов $\sum_{n=1}^{\infty} \alpha_{n}^{+}$ или $\sum_{n=1}^{\infty} \alpha_{n}^{-}$при расходимости второго приводит к тому, что упомянутый выше предел оказьвается равньм 0 или соответственно $+\infty$. Наконец, если оба ряда расходятся, то при подходящем выборе $\eta_{n}=t_{n} \xi_{n}$ величина $J_{\varepsilon}$ не имеет определенного предела при $\varepsilon \rightarrow 0$.

Я признателен А. М. Стёпину за полезные замечания и внимание к этой работе.

Пользуясь случаем, поздравляю П. Л. Ульянова с семидесятилетием и благодарю за постоянную поддержку.

\section{§1. Обозначения и терминология}

Далее символом $X$ обозначается вещественное локально выпуклое пространство; топологически сопряженное к $X$ будем обозначать через $X^{*}$, а борелевскую $\sigma$-алгебру $X$ - через $\mathscr{B}(X)$. Лебеговское пополнение $\mathscr{B}(X)$ относительно меры $\mu$ на $\mathscr{B}(X)$ обозначается через $\mathscr{B}(X)_{\mu}$. Функции, измеримые относительно $\mathscr{B}(X)_{\mu}$, называются $\mu$-измеримыми.

Если $H$ - сепарабельное гильбертово пространство, то через $\mathscr{H}(H)$ обозначается пространство всех операторов Гильберта-Шмидта на $H$ с нормой

$$
\|A\|_{\mathscr{H}}:=\left(\sum_{n=1}^{\infty}\left|A e_{n}\right|_{H}^{2}\right)^{1 / 2},
$$

где $\left\{e_{n}\right\}$ - произвольный ортонормированный базис в $H$. Через $\mathscr{L}_{(1)}(H)$ обозначается класс ядерных операторов в $H$, т.е. операторов вида $A B$, где $A, B \in \mathscr{H}(H)$. Для всякого ядерного оператора $A$ и всякого ортонормированного базиса $\left\{e_{n}\right\}$ в $H$ величина

$$
\operatorname{trace} A:=\sum_{n=1}^{\infty}\left(A e_{n}, e_{n}\right)_{H}
$$


не зависит от $\left\{e_{n}\right\}$ и называется следом $A$. Если симметричный непрерывный линейньй оператор $A$ в $H$ таков, что указанный выше ряд сходится для всякого ортонормированного базиса, то этот оператор - ядерный.

Всюду ниже термин "гауссовская мера" означает радоновскую гауссовскую меру на $X$, т.е. такую вероятностную меру $\gamma$ на $\mathscr{B}(X)$, что для всякого $f \in X^{*}$ индуцированная мера $\gamma \circ f^{-1}$ на прямой является гауссовской (возможно вырожденной), а кроме того, для всякого борелевского множества $B \subset X$ и всякого $\varepsilon>0$ найдется такой компакт $K \subset B$, что $\gamma(B \backslash K)<\varepsilon$. Впрочем, это дополнительное условие радоновости автоматически вьполнено для большинства пространств, встречающихся в приложениях (например, сепарабельных банаховых или сепарабельных пространств Фреше). Более того, без ушерба для обшности читатель может считать, что гауссовская мера $\gamma$, фигурируюшая в формулировках ниже, является либо стандартной винеровской мерой, либо счетным произведением стандартных одномерных гауссовских мер на счетном произведении прямых $\mathbb{R}^{\infty}$. Приводимые ниже результаты остаются в силе и без предположения о радоновости гауссовских мер на локально выпуклых пространствах, если ограничиться рассмотрением счетно-порожденных $\sigma$-алгебр. Основные сведения о гауссовских мерах можно найти в [5] и [8].

Средним гауссовской меры $\gamma$ на $X$ называется такой вектор $a_{\gamma} \in X$, что

$$
f\left(a_{\gamma}\right)=\int_{X} f(x) \gamma(d x) \quad \forall f \in X^{*}
$$

Гауссовская мера $\gamma$ на $X$ назьвается центрированной, если для всякого $f \in X^{*}$ мера $\gamma \circ f^{-1}$ является центрированной. Это равносильно тому, что $a_{\gamma}=0$. Для всякой гауссовской случайной величины $\xi$ будем обозначать через $\sigma(\xi)^{2}$ ее дисперсию, т.е. $\sigma(\xi)^{2}=\mathrm{E}|\xi-\mathrm{E} \xi|^{2}$, где $\mathrm{E}$ - математическое ожидание. Для центрированной гауссовской меры $\gamma$ через $X_{\gamma}^{*}$ обозначается замыкание $X^{*}$ в $L^{2}(\gamma)$. Элементы $X_{\gamma}^{*}$ называются $\gamma$-измеримьми линейными функционалами.

Пространство Камерона-Мартина $H(\gamma)$ гауссовской меры $\gamma$ на $X$ определяется как совокупность всех таких векторов $h \in X$, что мера $\gamma_{h}$, определяемая равенством $\gamma_{h}(B)=\gamma(B-h)$, эквивалентна $\gamma$. Пусть всюду ниже $\gamma$ - центрированная гауссовская мера. Известно, что

$$
H(\gamma)=\left\{x \in X:|h|_{H}<\infty\right\}
$$

где

$$
|h|_{H}=\sup \left\{f(h): f \in X^{*},\|f\|_{L^{2}(\gamma)} \leqslant 1\right\} .
$$

Кроме того, для каждого $h \in H(\gamma)$ сушествует единственньй элемент $\widehat{h} \in X_{\gamma}^{*}$, для которого

$$
f(h)=(f, \widehat{h})_{L^{2}(\gamma)} \quad \forall f \in X^{*} .
$$

При этом $|h|_{H}=\|\widehat{h}\|_{L^{2}(\gamma)}$. Пространство $H=H(\gamma)$ со скалярным произведением

$$
(h, k)_{H}=(\widehat{h}, \widehat{k})_{L^{2}(\gamma)}
$$


оказывается сепарабельным гильбертовым пространством, единичный шар которого компактен в $X$. Отображение $h \mapsto \widehat{h}$ является изометрией $H(\gamma)$ и $X_{\gamma}^{*}$. Обратное отображение $R_{\gamma}: X_{\gamma}^{*} \rightarrow H(\gamma)$ называется ковариационным оператором меры $\gamma$. Отметим, что $R_{\gamma}$ можно определить посредством равенства

$$
f\left(R_{\gamma} g\right)=\int_{X} f(x) g(x) \gamma(d x), \quad f \in X^{*}, \quad g \in X_{\gamma}^{*} .
$$

Каждый $\gamma$-измеримьй линейный функционал $g$ обладает собственно линейной (т.е. линейной в обычном смысле) модификацией $g_{0}$; при этом

$$
g_{0}(k)=\left(R_{\gamma} g, k\right)_{H} \quad \forall k \in H .
$$

Функция

$$
f \mapsto \sigma(f)^{2}=f\left(R_{\gamma} f\right)=\int_{X} f(x)^{2} \gamma(d x)
$$

на $X^{*}$ назьвается ковариацией мерь $\gamma$.

Отметим, что элемент $\widehat{h}$ называется измеримым линейным продолжением функционала $k \mapsto(k, h)_{H}$ на $H$. Известно, что всякий непрерьвный линейный оператор $A$ в $H$ обладает (единственньм с точностью до эквивалентной модификации) измеримым линейным продолжением $\widehat{A}$, т.е. $\widehat{A}$ - определенное на линейном подпространстве полной меры линейное отображение, совпадающее с $A$ на $H$, измеримое относительно пары $\sigma$-алгебр $\mathscr{B}(X)_{\gamma}$ и $\mathscr{B}(X)$ и обладающее тем свойством, что индуцированная мера $\gamma \circ \widehat{A}^{-1}$ - радоновская гауссовская. Если $A$ - оператор Гильберта-Шмидта (и только в этом случае), то $\widehat{A}$ обладает версией, принимающей значения в $H$.

Один из важнейших результатов теории гауссовских мер состоит в том, что образ меры $\gamma$ при отображении $\widehat{A}$ является мерой, эквивалентной $\gamma$, в точности тогда, ког да $A A^{*}-I$ есть оператор Гильберта-Шмидта и $A(H)=H$. При этом всякая центрированная гауссовская мера $\mu$ на $X$, эквивалентная $\gamma$, имеет такой вид. Точнее говоря, $\mu=\gamma \circ T^{-1}$, где $T$ - измеримое линейное продолжение оператора вида $I+K$, где $K$ - оператор Гильберта-Шмидта на $H$, не имеющий собственного числа - 1 . Плотность Радона-Никодима гауссовской меры $\nu$ на $X$, эквивалентной мере $\gamma$, имеет вид

$$
\frac{d \nu}{d \gamma}=\exp Q
$$

где $Q-\gamma$-измеримый многочлен второго порядка, т.е. элемент пространства $\mathscr{P}_{2}(\gamma)$, определяемого как замыкание в $L^{2}(\gamma)$ совокупности многочленов вида $Q_{n}\left(f_{1}, \ldots, f_{n}\right)$, где $Q_{n}$-многочлен второго порядкана $\mathbb{R}^{n}$ и $f_{i} \in X^{*}$. Аналогичным образом вводятся классы $\mathscr{P}_{k}(\gamma) \quad \gamma$-измеримых многочленов степени $k$ как замыкания в $L^{2}(\gamma)$ совокупности многочленов степени $k$ от элементов $X^{*}$. Известно, что то же самое множество получается, если вместо замькания в $L^{2}(\gamma)$ брать замыкание в смысле сходимости по мере $\gamma$. Пространство $\mathscr{P}_{k}(\gamma)$ можно представить в виде прямой суммы взаимно ортогональных подпространств $\mathscr{X}_{0}, \ldots, \mathscr{X}_{k}$. При этом $\mathscr{X}_{k}$ определяется как ортогональное дополнение к $\mathscr{P}_{k-1}(\gamma)$ в $\mathscr{P}_{k}(\gamma)$. В этих обозначениях $\mathscr{X}_{0}$ - одномерное пространство констант, $\mathscr{X}_{1}=X_{\gamma}^{*}$. Ортогональные проекторы на $\mathscr{X}_{k}$ обозначаются через $I_{k}$. Известно, что, хотя в определении измеримых многочленов участвует топологическое сопряженное, 
фактически классы $\mathscr{X}_{\boldsymbol{k}}$ не зависят от топологии в следующем смысле: если $X$ линейно и непрерывно вложено в локально выпуклое пространство $Y$, а $\gamma$ рассматривается как мера на $Y$, то классы $\mathscr{X}_{k}$ при этом не меняются.

Классы $\mathscr{X}_{k}$ входят в соболевские классы $W^{2, r}(\gamma)$ относительно меры $\gamma$ (см. $\left[8\right.$, гл. 5]); в частности, для их элементов определены производные $D_{H} F$ и $D_{H}^{2} F$ вдоль $H=H(\gamma)$. Например, если последовательность многочленов $F_{j}$ степени $k$ от непрерывных линейных функционалов сходится в $L^{2}(\gamma)$ (или хотя бы по мере $\gamma$ ) к $F$, то производные $\Phi$ реше функций $F_{j}$ вдоль $H$ (обозначаемые через $D_{H} F_{j}$ ) сходятся в пространстве $L^{2}(\gamma, H) \quad H$-значных отображений к некоторому отображению, обозначаемому через $D_{H} F$. Кроме того, вторые производные $D_{H}^{2} F_{j}$ сходятся в $L^{2}(\gamma, \mathscr{H}(H))$ к $\mathscr{H}(H)$-значному отображению $D_{H}^{2} F$. Если $k=2$, то $D_{H}^{2} F$ почти всюду совпадает с некоторым постоянньм оператором Гильберта-Шмидта на $H$.

Функция $q$ на $X$ называется $\gamma$-измеримой полунормой, если она $\gamma$-измерима и является полунормой в обьчном смысле на некотором линейном подпространстве полной $\gamma$-меры. Если при этом ограничение $q$ на $H(\gamma)$ является нормой, то $q$ называется $\gamma$-измеримой нормой. Как показано в [13] (см. также [8, предложение 4.3.3]), для всякой $\gamma$-измеримой полунормы $q$ сушествуют такие элементы $f_{n} \in X_{\gamma}^{*}$ и числа $\theta_{n} \geqslant 0$, что

$$
q(x)=\sup _{n}\left[f_{n}(x)+\theta_{n}\right] \quad \gamma \text {-п.в. }
$$

Ниже мы будем считать, что в этом равенстве измеримые линейные функционалы $f_{n}$ представлены своими собственно линейньми версиями (т.е. линейными на всем пространстве в обычном смысле). Мы будем рассматривать $\gamma$-измеримые нормы, удовлетворяющие условию

$$
\gamma(x \in X: q(x)<\varepsilon)>0 \quad \forall \varepsilon>0
$$

Это условие естественно при рассмотрении отношения в (0.1); если оно не вьполнено, то можно считать соответствуюшее отношение равным 1 при всех достаточно малых $\varepsilon$, что делает задачу тривиальной. При выполнении условия (1.3) в представлении (1.2) имеем $\theta_{n} \equiv 0$. Действительно, для каждого $n$ имеем $f_{n}(x)+\theta_{n} \leqslant$ $q(x)$ почти всюду, откуда в силу симметричности $\gamma$ получаем $f_{n}(-x)+\theta_{n} \leqslant q(x)$, т.е. $\theta_{n} \leqslant q(x)$ почти всюду.

\section{§2. Вспомогательные результаты}

В этом параграфе собраны вспомогательные результаты. Первые три леммы фактически хорошо известны, но для того чтобы пользоваться ими в приводимых формулировках, они снабжены доказательствами.

Лемма 2.1. Для всякого $\gamma$-измеримого многочлена второй степени $Q$ существуют ортонормированный базис $\left\{e_{n}\right\}$ в $H=H(\gamma)$ и вещественные числа $c, c_{n}, \alpha_{n}$ maкuе, что

$$
\begin{gathered}
\sum_{n=1}^{\infty} c_{n}^{2}<\infty, \quad \sum_{n=1}^{\infty} \alpha_{n}^{2}<\infty \\
Q(x)=c+\sum_{n=1}^{\infty} c_{n} \widehat{e}_{n}(x)+\sum_{n=1}^{\infty} \alpha_{n}\left(\widehat{e}_{n}(x)^{2}-1\right) \quad \gamma-n . \text { в. }
\end{gathered}
$$


где оба ряда сходятся почти всюду и во всех $L^{p}(\gamma)$. Обратно, всякая функиия $Q$ указанного вида представляет собой $\gamma$-измеримый многочлен второго порядка.

ДОКАЗАТЕЛЬСТВО. Отметим, что для всякого ортонормированного базиса $\left\{e_{n}\right\}$ в $H$ функции $\widehat{e}_{n}-$ независимые стандартные гауссовские случайные величины на $(X, \gamma)$. Поэтому при любом выборе чисел, удовлетворяющих условию (2.1), ряды в (2.2) сходятся почти всюду. Это вытекает, например, из теоремы Колмогорова-Хинчина $[14$, гл. IV,$\S 2$, теорема 1]. Тогда имеет место и сходимость во всех $L^{p}(\gamma)$ (см. [8, следствие 5.6.5]). Этим доказана вторая часть леммы. Пусть теперь $Q-\gamma$-измеримый многочлен второй степени. Тогда его вторая производная $D_{H}^{2} Q$ вдоль $H$ в смысле соболевского класса $W^{2,1}(\gamma)$ (см. [8, гл. 5]) совпадает $\gamma$-п.в. с некоторым неслучайньм симметричньм оператором Гильберта-Шмидта $K$ в $H$. По теореме Гильберта-Шмидта найдется такой ортонормированный базис $\left\{e_{n}\right\}$ в $H$, что $K e_{n}=2 \alpha_{n} e_{n}$. Как отмечено выше, функция

$$
G=\sum_{n=1}^{\infty} \alpha_{n}\left(\widehat{e}_{n}^{2}-1\right)
$$

корректно определена и является $\gamma$-измеримым многочленом второго порядка. При этом $D_{H}^{2}(Q-G)=0$, откуда вытекает, что $Q-G \in \mathscr{X}_{0} \oplus \mathscr{X}_{1}$. Действительно, функция $I_{2}(Q-G)$ разлагается в ряд из взаимно ортогональных функций вида $c\left(\widehat{e}_{i}-1\right)\left(\widehat{e}_{j}-1\right)$. Поэтому равенство $D_{H}^{2} I_{2}(Q-G)=0$ возможно лишь в случае, когда все элементы этого разложения равны нулю. Остается воспользоваться тем, что всякий элемент $g \in \mathscr{X}_{0} \oplus \mathscr{X}_{1}$ имеет вид

$$
g=c+\sum_{n=1}^{\infty} c_{n} \widehat{e}_{n} \quad \gamma \text {-п.в. }
$$

для некоторого $c \in \mathbb{R}^{1}$ и некоторой числовой последовательности $\left(c_{n}\right) \in l^{2}$.

Пусть $\left\{\xi_{n}\right\}$ - последовательность независимых стандартных гауссовских случайных величин, а случайная величина $Q$ определена равенством (0.2). Тогда случайная величина $\exp Q$ интегрируема в точности тогда, когда $\alpha_{n}<1 / 2$ для всех $n$. При этом

$$
\begin{aligned}
\mathrm{E}\left(\operatorname { e x p } \left(c+\sum_{n=1}^{\infty} c_{n} \xi_{n}+\sum_{n=1}^{\infty}\right.\right. & \left.\left.\alpha_{n}\left(\xi_{n}^{2}-1\right)\right)\right) \\
& =\exp \left(c+\frac{1}{2} \sum_{n=1}^{\infty} \frac{c_{n}^{2}}{1-2 \alpha_{n}}\right) \prod_{n=1}^{\infty} \frac{\exp \left(-\alpha_{n}\right)}{\sqrt{1-2 \alpha_{n}}}
\end{aligned}
$$

Это проверяется непосредственными вычислениями с учетом того факта, что произведение в правой части (2.3) сходится при $\sum_{n=1}^{\infty} \alpha_{n}^{2}<\infty$ и $\alpha_{n}<1 / 2$.

ЛЕмма 2.2. Пусть $\gamma-$ гауссовская мера на $X$, a $Q-$ такой $\gamma$-измеримый многочлен второго порядка, что $\exp Q \in L^{1}(\gamma)$. Тогда мера

$$
\mu=\left(\int_{X} \exp Q d \gamma\right)^{-1} \exp Q \cdot \gamma
$$

является гауссовской. 
ДоКАЗАТЕЛЬСтво. Как вытекает из леммы 2.1 и соотношения (2.3), сушествует такое $p>1$, что $\exp Q \in L^{p}(\gamma)$. Возьмем произвольный ортонормированный базис $\left\{e_{n}\right\}$ в $H(\gamma)$. Пусть $Q_{n}$ - конечномерные приближения $Q$, построенные как условные математические ожидания относительно $\sigma$-алгебр, порожденных $\widehat{e}_{1}, \ldots, \widehat{e}_{n}$ (см. [8, следствие 3.4.5]). Тогда $Q_{n}-$ экспоненциально $\gamma$-интегрируемые многочлены второй степени от $\widehat{e}_{1}, \ldots, \widehat{e}_{n}$. Заметим, что многочлен второй степени на $\mathbb{R}^{n}$ имеет вид $F(x)=(A x, x)+(x, v)+c$, где $A$ - симметричньй оператор, $v \in \mathbb{R}^{n}, c \in \mathbb{R}^{1}$. Такой многочлен экспоненциально интегрируем относительно стандартной гауссовской меры $\gamma_{n}$ в точности тогда, когда $A<I / 2$. Ясно, что в этом случае мера

$$
\|\exp F\|_{L^{1}\left(\gamma_{n}\right)}^{-1} \exp F \cdot \gamma_{n}
$$

- гауссовская. Это показывает, что меры

$$
\nu_{n}=\left\|\exp Q_{n}\right\|_{L^{1}(\gamma)}^{-1} \exp Q_{n} \cdot \gamma
$$

являются гауссовскими. Поскольку последовательность $\left\{Q_{n}\right\}$ сходится к $Q$ почти всюду, а последовательность $\left\{\exp Q_{n}\right\}$ равномерно интегрируема ввиду оценки

$$
\int_{X} \exp \left(p Q_{n}\right) d \gamma \leqslant \int_{X} \exp (p Q) d \gamma
$$

вытекающей из неравенства Йенсена для условных математических ожиданий, то меры $\nu_{n}$ сходятся к $\mu$ по вариации. Значит, $\mu$-гауссовская мера.

Очевидным образом верно и в некотором смысле обратное к доказанной лемме утверждение.

ЛЕмма 2.3. Если $\mu$ - гауссовская мера, эквивалентная $\gamma$, то ее плотность Радона-Никодима является $\gamma$-измеримым многочленом второго порядка и имеет вид (2.2).

ДокАЗАТЕльство. Пусть $\varrho=d \mu / d \gamma$. Беря, как и в предыдущей лемме, условные математические ожидания $\varrho_{n}$ функции $\varrho$ относительно $\sigma$-алгебр, порожденных $\widehat{e}_{1}, \ldots, \widehat{e}_{n}$, получаем, что $\varrho_{n} \rightarrow \varrho$ почти всюду. Положим $P_{n} x=\left(\widehat{e}_{1}, \ldots, \widehat{e}_{n}\right)$, $\mu_{n}=\mu \circ P_{n}^{-1}, \gamma_{n}=\gamma \circ P_{n}^{-1}$. Заметим, что $\varrho_{n}(x)=r_{n}\left(P_{n}(x)\right) \gamma$-п.в., где $r_{n}$ есть плотность меры $\mu_{n}$ относительно $\gamma_{n}$. Действительно, для всякой ограниченной борелевской функции $\varphi$ на $\mathbb{R}^{n}$ справедливо равенство

$$
\begin{aligned}
\int_{X} \varphi\left(P_{n} x\right) \varrho_{n}(x) \gamma(d x) & =\int_{X} \varphi\left(P_{n} x\right) \varrho(x) \gamma(d x)=\int_{X} \varphi\left(P_{n} x\right) \mu(d x) \\
& =\int_{\mathbb{R}^{n}} \varphi(y) \mu_{n}(d y)=\int_{\mathbb{R}^{n}} \varphi(y) r_{n}(y) \gamma_{n}(d y) \\
& =\int_{X} \varphi\left(P_{n} x\right) r_{n}\left(P_{n} x\right) \gamma(d x) .
\end{aligned}
$$

Поскольку в конечномерном случае наше утверждение очевидно, то $\varrho_{n}=\exp F_{n}$, где $F_{n}$-многочлен второго порядка от $\widehat{e}_{1}, \ldots, \widehat{e}_{n}$, т.е. $F_{n}-\gamma$-измеримый многочлен второго порядка. Последовательность $\left\{F_{n}\right\}$ сходится $\gamma$-почти всюду ввиду сходимости $\left\{\exp F_{n}\right\}$, поэтому ее предел есть также некоторый $\gamma$-измеримый многочлен второго порядка $F$, откуда $\varrho=\exp F$. 
Замечание 2.4. Отметим, что если функция $Q \in \mathscr{X}_{0} \oplus \mathscr{X}_{2}$ неотрицательна, то в (2.2) имеем

$$
\alpha_{n} \geqslant 0, \quad \sum_{n=1}^{\infty} \alpha_{n}<\infty, \quad c-\sum_{n=1}^{\infty} \alpha_{n} \geqslant 0 .
$$

Действительно, условное математическое ожидание $Q$ относительно $\sigma$-алгебры, порожденной $\widehat{e}_{1}, \ldots, \widehat{e}_{m}$, совпадает с $c+\sum_{n=1}^{m} \alpha_{n}\left(\widehat{e}_{n}^{2}-1\right)$ и неотрищательно, откуда утверждение вытекает немедленно, ибо многочлен

$$
c+\sum_{n=1}^{m} \alpha_{n}\left(x_{n}^{2}-1\right)
$$

на $\mathbb{R}^{m}$ почти всюду неотрищателен лишь в случае, когда числа $\alpha_{n}$ и $c-\sum_{n=1}^{m} \alpha_{n}$ неотрицательны. В частности, $D_{H}^{2} Q$ является ядерным оператором. Это показывает, что функция $Q \in \mathscr{X}_{0} \oplus \mathscr{X}_{2}$ с производной $D_{H}^{2} Q$, не являющейся ядерньм оператором, не может быть записана в виде $Q_{1}-Q_{2}$ с неотрицательньми $Q_{1}, Q_{2} \in \mathscr{X}_{0} \oplus \mathscr{X}_{2} ;$ в частности, так обстоит дело для $Q=\sum_{n=1}^{\infty} n^{-1}\left(\widehat{e}_{n}^{2}-1\right)$. Ясно, что условие $D_{H}^{2} Q \in \mathscr{L}_{(1)}(H)$ также и достаточно для существования разложения в разность неотрицательных многочленов второго порядка. Действительно, можно положить

$$
Q_{1}=I_{0}(Q)^{+}-\sum_{n=1}^{\infty} \alpha_{n}^{-}+\sum_{n=1}^{\infty} \alpha_{n}^{+} \xi_{n}^{2}
$$

где $\alpha_{n}^{+}$и $\alpha_{n}^{-}-$неотрицательные и отрицательные собственные числа оператора $D_{H}^{2} F$ в собственном базисе $\left\{e_{n}\right\}$ и $\xi_{n}=\widehat{e}_{n}$.

Важный частный случай квадратичных форм, допускающих разложение в разность неотрицательных измеримых квадратичных форм, - непрерывные формы.

Лемма 2.5. Пусть $Q$ - квадратичная форма на $X$ в обычном алгебраическом смысле, являющаяся секвенциально непрерывной (т.е. из сходимости $x_{n} \rightarrow x$ в $X$ вытекает сходимость $\left.Q\left(x_{n}\right) \kappa Q(x)\right)$. Тогда существуют такие числа $\alpha_{n}$ и ортонормированный базис $\left\{\xi_{n}\right\}$ в $X_{\gamma}^{*}$, что $\sum_{n=1}^{\infty}\left|\alpha_{n}\right|<\infty$ и $Q=\sum_{n=1}^{\infty} \alpha_{n} \xi_{n}^{2}$ п.в. При этом ограничение $Q$ на $H=H(\gamma)$ имеет вид $(S h, h)_{H}$, әде $S=2^{-1} D_{H}^{2} Q$ - ядерный оператор в $H$ с собственными числами $\alpha_{n}$, и справедливо равенство

$$
\int_{X} Q(x) \gamma(d x)=\frac{1}{2} \operatorname{trace} D_{H}^{2} Q
$$

ДоказАТЕльство. Сушествует симметричная билинейная функция $\Psi$ на $X$, для которой $Q(x)=\Psi(x, x)$. Пусть $\left\{e_{n}\right\}$ - ортонормированньй базис в $H$. Тогда $\lim _{m \rightarrow \infty} \sum_{n=1}^{m} \widehat{e}_{n}(x) e_{n}=x$ для $\gamma$-п.в. $x \in X$ (см. [8, теорема 3.4.4]). В силу секвенциальной непрерывности $Q$ получаем

$$
Q(x)=\lim _{m \rightarrow \infty} Q\left(\sum_{n=1}^{m} \widehat{e}_{n}(x) e_{n}\right) \text { п.в. }
$$

Ясно, что

$$
Q\left(\sum_{n=1}^{m} \widehat{e}_{n}(x) e_{n}\right)=\sum_{n=1}^{m} Q\left(e_{n}\right) \widehat{e}_{n}(x)^{2}+2 \sum_{n \neq k, n, k \leqslant m} \Psi\left(e_{n}, e_{k}\right) \widehat{e}_{n}(x) \widehat{e}_{k}(x) .
$$


Следовательно, квадратичные формы в (2.5) сходятся не только почти всюду, но и в $L^{2}(\gamma)$ (см. $\left.\S 1\right)$, причем $Q \in \mathscr{P}_{2}(\gamma)$ и $D_{H}^{2} Q$ - симметричный оператор ГильбертаШмидта в $H$, который мы обозначим через $2 S$. Пусть теперь $\left\{e_{n}\right\}-$ собственный базис $S$. Тогда $Q(x)=\sum_{n=1}^{\infty} Q\left(e_{n}\right) \widehat{e}_{n}(x)^{2}$ п.в. Интегрируя это равенство с учетом соотношения

$$
\left(2 S e_{n}, e_{n}\right)_{H}=\partial_{e_{n}}^{2} Q=2 Q\left(e_{n}\right),
$$

получаем (2.4). Заметим, что $S \in \mathscr{L}_{(1)}(H)$, ибо значение $\sum_{n=1}^{\infty}\left(S e_{n}, e_{n}\right)_{H}$ - одно и то же для всех перестановок $\left\{e_{n}\right\}$.

Следует подчеркнуть, что не всякая непрерьвная квадратичная форма $Q$ на банаховом пространстве (в отличие от случая гильбертова пространства) может быть разложена в разность неотрицательных непрерывных квадратичных форм. Возьмем, например, последовательность таких норм $q_{n}$ на плоскости, что форма $K(x, y)=x^{2}-y^{2}$ ограничена по модулю единицей на единичном шаре $U_{n}$ по норме $q_{n}$, а ее положительная часть принимает на $U_{n}$ значение $4^{n}$ (в качестве $U_{n}$ можно взять эллипсоиды с сильно вытянутыми осями вдоль биссектрисы первого и третьего квадрантов и сильно сжатыми осями вдоль биссектрисы второго и четвертого квадрантов). Возьмем в качестве $X$ пространство последовательностей $z=\left(x_{n}, y_{n}\right)$ с нормой $\|z\|=\sup _{n} q_{n}\left(\left(x_{n}, y_{n}\right)\right)$, а в качестве $Q$ форму $\sum_{n=1}^{\infty} 2^{-n}\left(x_{n}^{2}-y_{n}^{2}\right)$. Тогда $Q$ нельзя разложить в разность неотрицательных непрерывных квадратичных форм. Более короткий, но менее наглядный пример можно извлечь из [15, упражнение 3г, с. 138]: возьмем рефлексивное банахово пространство $Y$, не являюшееся линейно гомеоморфньм гильбертову пространству, и на пространстве $Y \times Y^{*}$ положим $Q\left(y, y^{*}\right)=y^{*}(y)$.

Из сказанного выше явствует, что задача изучения предела в (0.1) равносильна задаче исследования предела

$$
\lim _{\varepsilon \rightarrow 0} \frac{1}{\gamma(\{q \leqslant \varepsilon\})} \int_{q \leqslant \varepsilon} \exp Q d \gamma
$$

для центрированной гауссовской меры $\gamma$ и $\gamma$-измеримого многочлена второго порядка $Q$ (или, что также равносильно, случайной величины вида $(0.2))$.

\section{§3. Основные результаты}

ЛЕмма 3.1. Пусть $\gamma-$ центрированная гауссовская мера на $X, V-\gamma-и з м е-$ римое выпуклое симметричное множество положительной меры, а функиии $Q_{1}, Q_{2} \in \mathscr{X}_{0} \oplus \mathscr{X}_{2}$ неотрицательнь. Тогда

$$
\int_{X} \exp \left(-Q_{2}\right) d \gamma \leqslant \frac{1}{\gamma(V)} \int_{V} \exp \left(Q_{1}-Q_{2}\right) d \gamma \leqslant \int_{X} \exp Q_{1} d \gamma
$$

ДокАЗАТЕльство. Поскольку $\exp \left(-Q_{2}\right) \leqslant \exp \left(Q_{1}-Q_{2}\right) \leqslant \exp Q_{1}$, то достаточно рассмотреть отдельно случаи, когда $Q_{1}=0$ и $Q_{2}=0$. Пусть $Q_{2}=0$. Мы знаем, что

$$
Q_{1}=c+\sum_{n=1}^{\infty} \alpha_{n} \xi_{n}^{2}
$$


где

$$
c \geqslant 0, \quad \alpha_{n} \geqslant 0, \quad \sum_{n=1}^{\infty} \alpha_{n}<\infty
$$

a $\left\{\xi_{n}\right\}$ - ортонормированный базис в $X_{\gamma}^{*}$. Ясно, что можно считать число $c$ равньм нулю, а функцию ехр $Q_{1}$ интегрируемой (в противном случае утверждение тривиально). Мера

$$
\nu:=\left\|\exp Q_{1}\right\|_{L^{1}(\gamma)}^{-1} \exp Q_{1} \cdot \gamma
$$

является центрированной гауссовской ввиду леммы 2.2 и ее ковариация мажорирует ковариацию меры $\gamma$. Действительно, пусть $f \in X^{*}$. Тогда $f=\sum_{n=1}^{\infty} c_{n} \xi_{n}$, где $\left(c_{n}\right) \in l^{2}$. Интегралы $f^{2}$ относительно мер $\nu$ и $\gamma$ равны суммам соответствуюших интегралов от $c_{n}^{2} \xi_{n}^{2}$ ввиду независимости случайных величин $\xi_{n}$. Следовательно, утверждение сводится к одномерному случаю, в котором оно очевидно. Значит, $\nu(V) \leqslant \gamma(V)$ (см. [8, теорема 3.3.5]), что и утверждалось. Случай $Q_{1}=0$ аналогичен.

СЛЕДСТВИЕ 3.2. Пусть $\gamma-$ иентрированная гауссовская мера на $X, V$ $\gamma$-измеримое выпуклое симметричное множество положительной меры $и$ пусть $Q=\sum_{n=1}^{\infty} \alpha_{n}\left(\xi_{n}^{2}-1\right)$, где $\alpha_{n} \geqslant 0, \sum_{n=1}^{\infty} \alpha_{n}^{2}<\infty, u\left\{\xi_{n}\right\}-$ ортонормированная последовательность в $X_{\gamma}^{*}$. Тогда

$$
\frac{1}{\gamma(V)} \int_{V} \exp Q d \gamma \leqslant \int_{X} \exp Q d \gamma
$$

ДоказАтЕльство. Достаточно рассмотреть случай, когда функция $\exp Q$ интегрируема, что соответствует условию $\alpha_{n}<1 / 2$ для всех $n$. Если $Q$ заменить на $Q_{k}=\sum_{n=1}^{k} \alpha_{n}\left(\xi_{n}^{2}-1\right)$, то утверждение верно по предыдущей лемме. Теперь остается заметить, что $\exp Q_{k} \rightarrow \exp Q$ в $L^{1}(\gamma)$. Действительно, последовательность $\exp Q_{k}$ сходится почти всюду к $\exp Q$ и равномерно интегрируема, поскольку сушествует такое число $p>1$, что $\sup _{k}\left\|\exp Q_{k}\right\|_{L^{p}(\gamma)}<\infty$.

ТЕОРема 3.3. Пусть $\gamma-$ центрированная гауссовская мера на $X, q-\gamma$-измеримая норма и пусть функиия $Q \in \mathscr{P}_{2}$ такова, что $D_{H}^{2} Q-$ ядерный оператор (что равносильно существованию разложсения $Q=f+Q_{1}-Q_{2}$, әде $f \in \mathscr{X}_{1}$ и $Q_{1}, Q_{2} \in \mathscr{X}_{0} \oplus \mathscr{X}_{2}$ неотрицательнь). Предположсим, что функция $\exp Q$ интегрируема. Тогда

$$
\lim _{\varepsilon \rightarrow 0} \frac{1}{\gamma\left(V_{\varepsilon}\right)} \int_{V_{\varepsilon}} \exp Q(x) \gamma(d x)=\exp \left(I_{0}(Q)-\frac{1}{2} \operatorname{trace} D_{H}^{2} Q\right),
$$

əде $V_{\varepsilon}=\{x: q(x) \leqslant \varepsilon\}$.

ДокАзАтЕльство. Функция $Q$ имеет представление (2.2). Можно считать, что $I_{0}(Q)-\frac{1}{2} \operatorname{trace} D_{H}^{2} Q=0$. Положим

$$
Q_{1}=\sum_{n=1}^{\infty} \alpha_{n}^{+} \widehat{e}_{n}^{2}, \quad Q_{2}=\sum_{n=1}^{\infty} \alpha_{n}^{-} \widehat{e}_{n}^{2},
$$

где $\alpha_{n}^{+}$и $\alpha_{n}^{-}-$положительные и отрицательные собственные числа $D_{H}^{2} Q / 2$ соответственно, причем $\alpha_{n}^{+} \alpha_{n}^{-}=0$ для всех $n$. Предположим сначала, что $f=0$ и

$$
\exp \left(8 Q_{1}\right) \in L^{1}(\gamma)
$$


Из условия вытекает, что $q(x)=\sup _{n} f_{n}(x)$ п.в., где $\left\{f_{n}\right\} \subset X_{\gamma}^{*}$ имеет плотную линейную оболочку в $X_{\gamma}^{*}$. В самом деле, если линейная оболочка $\left\{f_{n}\right\}$ не является плотной в $X_{\gamma}^{*}$, то найдется нетривиальный вектор $h \in H(\gamma)$ с $f_{n}(h)=0$ для всех $n$, поскольку $f_{n}-$ собственно линейные функционалы, а тогда $q(h)=0$. Легко видеть, что наше утверждение верно, если $Q_{1}$ и $Q_{2}$ являются конечными суммами квадратов конечных линейных комбинаций функционалов $f_{n}$. Действительно, в этом случае $Q_{1} \leqslant c q^{2}$ и $Q_{2} \leqslant c q^{2}$ для некоторого $c>0$ ввиду неравенства $\left|f_{n}\right| \leqslant q$, поэтому $\exp \left(-2 c \varepsilon^{2}\right) \leqslant \exp \left(Q_{1}-Q_{2}\right) \leqslant \exp \left(2 c \varepsilon^{2}\right)$ на множестве $\{q \leqslant \varepsilon\}$. Кроме того, общий случай сводится к случаю $Q_{2}=0$. В самом деле, полагая

$$
J_{\varepsilon}(\gamma, Q)=\frac{1}{\gamma\left(V_{\varepsilon}\right)} \int_{V_{\varepsilon}} \exp Q d \gamma
$$

и вводя центрированные гауссовские меры

$$
\nu:=\left\|\exp \left(-Q_{2}\right)\right\|_{L^{1}(\gamma)}^{-1} \exp \left(-Q_{2}\right) \cdot \gamma
$$

получаем

$$
J_{\varepsilon}\left(\gamma, Q_{1}-Q_{2}\right)=\frac{J_{\varepsilon}\left(\nu, Q_{1}\right)}{J_{\varepsilon}\left(\nu, Q_{2}\right)}
$$

поскольку

$$
\gamma\left(V_{\varepsilon}\right)=\int_{V_{\varepsilon}} \exp \left(Q_{2}\right) d \nu \int_{X} \exp \left(-Q_{2}\right) d \gamma
$$

Заметим, что случайные величины $\xi_{n}$ ортогональны в $L^{2}(\nu)$. Кроме того,

$$
\left\|\xi_{n}\right\|_{L^{2}(\nu)}^{2}=\left(1+2 \beta_{n}\right)^{-1} .
$$

Поэтому $Q_{2}=\sum_{n=1}^{\infty} \beta_{n}\left(1+2 \beta_{n}\right)^{-1} \zeta_{n}^{2}$, где $\left\{\zeta_{n}\right\}-$ ортонормированный базис в $X_{\nu}^{*}$, т.е. $Q_{2}$ удовлетворяет исходным условиям и относительно меры $\nu$.

Итак, обратимся к случаю, когда

$$
Q_{1}=\sum_{n=1}^{\infty} \alpha_{n} \xi_{n}^{2}
$$

где $\alpha_{n} \geqslant 0, \sum_{n=1}^{\infty} \alpha_{n}<\infty$ и $\left\{\xi_{n}\right\}$ - ортонормированная последовательность в $X_{\gamma}^{*}$. Пусть $S_{n}=\sum_{i=1}^{n} \alpha_{i} \xi_{i}^{2}$. Зафиксируем $q>1$. По теореме Лебега сушествует такое $n$, что

$$
\int_{X} \exp \left(2 Q_{1}-2 S_{n}\right) d \gamma \leqslant q^{2}
$$

Используя лемму 3.1 и тот факт, что $Q_{1}-S_{n} \geqslant 0$, получаем оценку

$$
\begin{aligned}
1 & \leqslant J_{\varepsilon}\left(\gamma, Q_{1}\right)=J_{\varepsilon}\left(\gamma, Q_{1}-S_{n}+S_{n}\right)=\frac{1}{\gamma\left(V_{\varepsilon}\right)} \int_{V_{\varepsilon}} \exp S_{n} \exp \left(Q_{1}-S_{n}\right) d \gamma \\
& \leqslant \sqrt{J_{\varepsilon}\left(\gamma, 2 S_{n}\right)} \sqrt{J_{\varepsilon}\left(\gamma, 2 Q_{1}-2 S_{n}\right)} \\
& \leqslant \sqrt{J_{\varepsilon}\left(\gamma, 2 S_{n}\right)} \sqrt{\int_{X} \exp \left(2 Q_{1}-2 S_{n}\right) d \gamma} \leqslant q \sqrt{J_{\varepsilon}\left(\gamma, 2 S_{n}\right)} .
\end{aligned}
$$


В силу произвольности $q>1$ эта оценка показьвает, что достаточно доказать, что $\lim _{\varepsilon \rightarrow 0} J_{\varepsilon}\left(\gamma, 2 S_{n}\right)=1$. По индукции это сводится к случаю $\alpha_{1}=\cdots=\alpha_{n-1}=0$, поскольку мера $\nu:=\left\|\exp \left(2 S_{n-1}\right)\right\|_{L^{1}(\gamma)}^{-1} \exp \left(2 S_{n-1}\right) \cdot \gamma$ - центрированная гауссовская и

$$
J_{\varepsilon}\left(\gamma, 2 S_{n}\right)=J_{\varepsilon}\left(\nu, 2 \alpha_{n} \xi_{n}^{2}\right) J_{\varepsilon}\left(\gamma, 2 S_{n-1}\right) .
$$

Положим $\xi=\sqrt{2 \alpha_{n}} \xi_{n}$. Тогда $\sigma(\xi)^{2}<1 / 8$ в силу интегрируемости $\exp \left(8 Q_{1}\right)$. Опять зафиксируем $q>1$. Найдем такой функционал $\eta$ из линейной оболочки $\left\{f_{n}\right\}$, что

$$
\sigma(\xi-\eta)^{2}<\frac{q^{2}-1}{8 q^{2}}
$$

Затем найдем такое $\varepsilon_{1}>0$, что

$$
J_{\varepsilon}\left(\gamma, 4 \eta^{2}\right) \leqslant q \quad \forall \varepsilon \in\left(0, \varepsilon_{1}\right) .
$$

Поскольку $\xi^{2} \leqslant 2(\xi-\eta)^{2}+2 \eta^{2}$, то при $\varepsilon \in\left(0, \varepsilon_{1}\right)$ в силу оценки $(3.2)$ получаем

$$
\begin{aligned}
1 & \leqslant J_{\varepsilon}\left(\gamma, \xi^{2}\right) \leqslant J_{\varepsilon}\left(\gamma, 2(\xi-\eta)^{2}+2 \eta^{2}\right) \leqslant \sqrt{J_{\varepsilon}\left(\gamma, 4(\xi-\eta)^{2}\right)} \sqrt{J_{\varepsilon}\left(\gamma, 4 \eta^{2}\right)} \\
& \leqslant \sqrt{q \int_{X} \exp \left(4(\xi-\eta)^{2}\right) d \gamma} \leqslant\left(1-8 \sigma(\xi-\eta)^{2}\right)^{-1 / 4} \sqrt{q} \leqslant q .
\end{aligned}
$$

В силу произвольности $q>1$ при сделанных выше дополнительных предположениях утверждение доказано.

Случай, когда $f \neq 0$, сводится к случаю, рассмотренному выше. Действительно, в силу неравенства Коши-Буняковского имеем

$$
\begin{aligned}
\left|J_{\varepsilon}(\gamma, Q+f)-J_{\varepsilon}(\gamma, Q)\right| & =\left|\frac{1}{\gamma\left(V_{\varepsilon}\right)} \int_{V_{\varepsilon}} \exp Q(\exp f-1) d \gamma\right| \\
& \leqslant \sqrt{J_{\varepsilon}(\gamma, 2 Q)} \sqrt{J_{\varepsilon}(\gamma, 2 f)-2 J_{\varepsilon}(\gamma, f)+1} \\
& \leqslant \sqrt{\int_{X} \exp 2 Q d \gamma} \sqrt{J_{\varepsilon}(\gamma, 2 f)-2 J_{\varepsilon}(\gamma, f)+1}
\end{aligned}
$$

Правая часть этой оценки стремится к нулю при стремлении $\varepsilon$ к нулю в силу [8, следствие 4.4.5].

Наконец, освободимся от предположения об интегрируемости $\exp \left(8 Q_{1}\right)$. Мы знаем, что найдется такое $\lambda>1$, что функция $\exp \left(\lambda Q_{1}\right) \gamma$-интегрируема. Функции $G(z, \varepsilon)=J_{\varepsilon}(\gamma, z Q)$ голоморфны по $z$ при $0<\operatorname{Re} z<\lambda$ и равномерно ограничены по $\varepsilon>0$ и $z$ с $0<\operatorname{Re} z<\lambda$ ввиду оценки (3.1). Поскольку они сходятся к 1 при $\varepsilon \rightarrow 0$ для всех достаточно малых действительных $z$, то сходимость имеет место для всех $z$ из $[0,1]$ ввиду теоремы Витали (см. [16, гл. V, §5.2]).

Отметим один случай, в котором для вывода о сушествовании предела в (0.1) не требуется изучать собственные числа соответствуюшей формы.

СлЕДСТВИЕ 3.4. Пусть $\gamma$ и $q-$ те же, что и в предыдущей теореме, $\alpha_{n}$ - вещественнье числа с $\sum_{n=1}^{\infty}\left|\alpha_{n}\right|<\infty, Q=\sum_{n=1}^{\infty} \alpha_{n} \zeta_{n}^{2}$, әде $\zeta_{n} \in X_{\gamma}^{*} u$ $\sigma\left(\zeta_{n}\right)^{2} \leqslant 1$. Тогда предел в (3.3) равен 1 . В частности, это верно для всякой секвенциально непрерывной квадратичной формы $Q$. 
ДокАЗАТЕЛЬСтво. Хотя первое утверждение можно усмотреть и из доказательства предыдущей теоремы, мы получим его из (3.3), проверив равенство (2.4) для нашей функции $Q$. Действительно, достаточно взять ортонормированный базис $\left\{e_{j}\right\}$ в $H$ и заметить, что

$$
\partial_{h}^{2}\left(\zeta_{n}^{2}\right)=2\left(\widehat{h}, \zeta_{n}\right)_{L^{2}(\gamma)}^{2}
$$

для всех $h \in H$. Поскольку $D_{H}^{2} Q-$ ядерный оператор в силу замечания 2.4, то $(2.4)$ вытекает из абсолютной сходимости двойного ряда

$$
\sum_{j=1}^{\infty} \sum_{n=1}^{\infty} \alpha_{n}\left(\widehat{e}_{j}, \zeta_{n}\right)_{L^{2}(\gamma)}^{2}=\sum_{n=1}^{\infty} \alpha_{n}\left\|\zeta_{n}\right\|_{L^{2}(\gamma)}^{2} .
$$

Второе утверждение непосредственно следует из леммы 2.5.

Комбинируя теорему 3.3 и следствие 3.2 , получаем, что

$$
\lim _{\varepsilon \rightarrow 0} J_{\varepsilon}(\gamma, Q)=0
$$

если $Q=Q_{1}+Q_{2}$, где $Q_{1}$ и $Q_{2}-\gamma$-измеримые многочлены второго порядка, такие, что $D_{H}^{2} Q_{1}$ - ядерный оператор, а оператор $D_{H}^{2} Q_{2}$ неотрицателен и имеет бесконечньй след. Действительно, в силу доказанного выше достаточно показать это для $Q_{1}=0$ и $Q_{2}=\sum_{n=1}^{\infty} \alpha_{n}\left(\xi_{n}^{2}-1\right)$, где

$$
\alpha_{n} \geqslant 0, \quad \sum_{n=1}^{\infty} \alpha_{n}^{2}<\infty \text { и } \sum_{n=1}^{\infty} \alpha_{n}=\infty .
$$

Положим $S_{k}=\sum_{n=1}^{k} \alpha_{n}\left(\xi_{n}^{2}-1\right)$. Предполагая, что функция $\exp \left(2 Q_{2}\right)$ интегрируема, ввиду следствия 3.2 и неравенства Коши-Буняковского получаем

$$
J_{\varepsilon}\left(\gamma, Q_{2}\right)^{2} \leqslant J_{\varepsilon}\left(\gamma, 2 S_{k}\right) J_{\varepsilon}\left(\gamma, 2 Q_{2}-2 S_{k}\right) \leqslant J_{\varepsilon}\left(\gamma, 2 S_{k}\right) \int_{X} \exp \left(2 Q_{2}\right) d \gamma .
$$

Первый сомножитель в правой части стремится к $\exp \left(-\sum_{n=1}^{k} \alpha_{n}\right)$ при $\varepsilon \rightarrow 0$, откуда и вытекает требуемое. Наконец, заметим, что без ущерба для общности можно считать функцию $\exp \left(2 Q_{2}\right)$ интегрируемой, поскольку $\alpha_{n} \rightarrow 0$ и при достаточно больших $k$ это предположение выполнено для $Q_{2}-S_{k}$ вместо $Q_{2}$ и меры $\left\|\exp S_{k}\right\|_{L^{1}(\gamma)}^{-1} \exp S_{k} \cdot \gamma$ вместо $\gamma$.

Рассматривая квадратичные формы $Q$ с неположительными операторами $D_{H}^{2} Q$ не следового класса, получаем бесконечность в качестве предела $J_{\varepsilon}(\gamma, Q)$. Эти рассуждения можно резюмировать следуюшим образом.

СлЕДСТвИЕ 3.5. Пусть $\gamma$ - центрированная гауссовская мера на $X$ и $Q \in \mathscr{P}_{2}$ - такой $\gamma$-измеримый многочлен второго порядка, что $D_{H}^{2} Q$ имеет суммируемый (возможно, $\kappa-\infty$ или $+\infty$ ) след

$$
\text { trace } D_{H}^{2} Q=2 \sum_{n=1}^{\infty} \alpha_{n}^{+}+2 \sum_{n=1}^{\infty} \alpha_{n}^{-}
$$

где $2 \alpha_{n}^{+}$и $2 \alpha_{n}^{-}$соответственно положительные и отрицательные собственные числа $D_{H}^{2} Q$, причем хотя бы один из этих двух рядов сходится $\kappa$ конечному числу. Тогда

$$
\lim _{\varepsilon \rightarrow 0} \frac{1}{\gamma\left(V_{\varepsilon}\right)} \int_{V_{\varepsilon}} \exp Q(x) \gamma(d x)=\exp \left(I_{0}(Q)-\frac{1}{2} \operatorname{trace} D_{H}^{2} Q\right) .
$$

Как мы сейчас увидим, условная сходимость ряда из собственных чисел $D_{H}^{2} Q$ не влечет существование предела $J_{\varepsilon}(\gamma, Q)$. 
ПримеР 3.6. Если

$$
Q=\sum_{n=1}^{\infty} \alpha_{n}^{+}\left(\xi_{n}^{2}-1\right)+\sum_{n=1}^{\infty} \alpha_{n}^{-}\left(\xi_{n}^{2}-1\right)
$$

где $\left(\alpha_{n}\right) \in l^{2}, \alpha_{n}^{+}>0, \alpha_{n}^{-}<0, \alpha_{n}^{+} \alpha_{n}^{-}=0$ для всех $n$ и

$$
\sum_{n=1}^{\infty} \alpha_{n}^{+}=\sum_{n=1}^{\infty}\left|\alpha_{n}^{-}\right|=\infty
$$

то для подходящей нормы $q$ не существует определенного предела (конечного или бесконечного) величин $J_{\varepsilon}(\gamma, Q)$. Более того, можно так подобрать $q$, что $J_{\varepsilon}(\gamma, Q)$ будет осциллировать между 0 и $\infty$.

ДоКАЗАТЕЛЬСТво. Для упрошения обозначений мы подробно рассмотрим лишь пример $\left\{\alpha_{n}\right\}$ и $q$, в котором имеются две различные предельные точки; в обшем случае используется модификация этой конструкции.

Последовательность $\left\{\alpha_{n}\right\}$ будет построена как последовательность конечных блоков $\alpha_{n, 1}, \ldots, \alpha_{n, 2 m(n)}$, где $m(n)=n^{2} 2^{n+2}, \alpha_{n, j}=n^{-1} 2^{-n-2}$ и $\alpha_{n, m(n)+j}=$ $-\alpha_{n, j}$ при $j=1, \ldots, m(n)$. Тогда $\sum_{j=1}^{m(n)} \alpha_{n, j}=n$ и $\sum_{j=1}^{2 m(n)} \alpha_{n, j}^{2}=2^{-n-1}$. Мы найдем две последовательности положительных чисел $\varepsilon_{n}<1$ и $c_{n, j}$, $j=1, \ldots, 2 m(n)$, таких, что $\varepsilon_{n}>\varepsilon_{n+1}, c_{n, j}>n m(n)$ (это условие нужно для того, чтобы получить конечную норму) и, полагая

$$
\begin{aligned}
J_{n}\left(\varepsilon, c_{n, 1}, \ldots,\right. & \left.c_{n, 2 m(n)}\right) \\
& =\prod_{j=1}^{2 m(n)} \frac{1}{\gamma_{1}\left(\left[-\varepsilon c_{n, j}, \varepsilon c_{n, j}\right]\right)} \int_{|t| \leqslant \varepsilon c_{n, j}} \exp \left(\alpha_{n, j} t^{2}-\alpha_{n, j}\right) p(t) d t
\end{aligned}
$$

где $\gamma_{1}$ - стандартная гауссовская мера на $\mathbb{R}^{1}$ с плотностью $p$, имеем

$$
\begin{gathered}
\left|J_{n}\left(\varepsilon_{i}, c_{n, 1}, \ldots, c_{n, 2 m(n)}\right)-\prod_{j=1}^{m(n)} \frac{1}{\sqrt{1-4 \alpha_{n, j}^{2}}}\right| \leqslant 2^{-n-1}, \quad i<2 n \\
J_{n}\left(\varepsilon_{2 n}, c_{n, 1}, \ldots, c_{n, 2 m(n)}\right)<2 M e^{-n} \\
\left|J_{n}\left(\varepsilon, c_{n, 1}, \ldots, c_{n, 2 m(n)}\right)-1\right| \leqslant 2^{-n-1} \quad \forall \varepsilon \in\left(0, \varepsilon_{2 n+1}\right),
\end{gathered}
$$

где

$$
M=\prod_{n=1}^{\infty} \prod_{j=1}^{m(n)} \sqrt{1+4 \alpha_{n, j}^{2}}
$$

Такие последовательности строятся индуктивно. Предположим, что мы уже построили $\varepsilon_{i}, i<2 n$, и $c_{k, i}, k<n$. Возьмем $R>n m(n)$ настолько большим, что для всякого $i<2 n$ справедливо неравенство

$$
\left|J_{n}\left(\varepsilon_{i}, t_{1}, \ldots, t_{n(m)}, s_{1}, \ldots, s_{m(n)}\right)-\prod_{j=1}^{m(n)} \frac{1}{\sqrt{1-4 \alpha_{n, j}^{2}}}\right| \leqslant 2^{-n-1} \quad \forall t_{i}, s_{i} \geqslant R .
$$


Это возможно ввиду (2.3) и равенства $\sum_{j=1}^{2 m(n)} \alpha_{n, j}=0$. Далее, найдутся такие $\delta \in\left(0, R^{-1}\right)$ и $r>\delta$, что при $\delta_{i} \equiv \delta$ и $r_{i} \equiv r$ имеем

$$
J_{n}\left(\varepsilon, \delta_{1}, \ldots, \delta_{m(n)}, r_{1}, \ldots, r_{m(n)}\right)<2 e^{-n} \prod_{j=1}^{m(n)} \sqrt{1+4 \alpha_{n, j}^{2}}<2 M e^{-n} .
$$

Это возможно, ибо произведение первых $m(n)$ сомножителей в выражении для $J_{n}$ стремится при $\delta \rightarrow 0$ к числу $\exp \left(-\sum_{j=1}^{m(n)} \alpha_{n, j}\right)$, равному $\exp (-n)$, а произведение остальных $m(n)$ сомножителей согласно $(2.3)$ стремится при $r \rightarrow+\infty \mathrm{K}$

$$
\prod_{j=1}^{m(n)} \frac{\exp \left(\alpha_{n, j}\right)}{\sqrt{1+2 \alpha_{n, j}}} \leqslant \prod_{j=1}^{m(n)} \sqrt{1+4 \alpha_{n, j}^{2}} .
$$

Положим

$$
\begin{gathered}
\varepsilon_{2 n}=\frac{\delta^{2} \varepsilon_{2 n-1}}{R}, \\
c_{n, j}=\frac{R}{\varepsilon_{2 n-1} \delta}, j=1, \ldots, m(n), \quad c_{n, j}=\frac{r R}{\delta^{2} \varepsilon_{2 n-1}}, j=m(n)+1, \ldots, 2 m(n) .
\end{gathered}
$$

При таком выборе имеем

$$
\varepsilon_{2 n} c_{n, j}=\delta \text { при } j \leqslant m(n), \quad \varepsilon_{2 n} c_{n, j}=r \text { при } m(n)<j \leqslant 2 m(n) .
$$

Наконец, возьмем $c \in\left(0, \varepsilon_{2 n}\right]$ достаточно малым, чтобы получить

$$
\left|J_{n}\left(\varepsilon, c_{n, 1}, \ldots, c_{n, 2 m(n)}\right)-1\right| \leqslant 2^{-n-1} \quad \forall \varepsilon \in(0, c] .
$$

Это возможно ввиду равенства $\sum_{j=1}^{2 m(n)} \alpha_{n, j}=0$. Остается положить $\varepsilon_{2 n+1}=c / 2$. Эта конструкция дает желаемый пример, если мы возьмем в качестве $\gamma$ счетное произведение стандартных гауссовских мер на $\mathbb{R}^{1}$ и положим

$$
Q(x)=\sum_{n=1}^{\infty} \alpha_{n}\left(x_{n}^{2}-1\right), \quad q(x)=\sup _{n}\left|x_{i} / \lambda_{i}\right|,
$$

где $\left\{\lambda_{i}\right\}$ - последовательность, образованная конечными блоками $c_{n, j}, j \leqslant 2 m(n)$. Отметим, что $q<\infty$ почти всюду и $\gamma(q<\varepsilon)>0 \forall \varepsilon>0$ в силу нашего выбора $\lambda_{i}$. Последовательность $J_{\varepsilon_{2 n}}(\gamma, Q)$ сходится к нулю, в то время как последовательность $J_{\varepsilon_{2 n+1}}(\gamma, Q)$ сходится к положительному числу. В общем случае при выполнении условия (3.5) можно считать числа $\alpha_{n}^{+}$и $\left|\alpha_{n}^{-}\right|$упорядоченными по убыванию и меньшими $1 / 2$. Выбрав подпоследовательности $n_{k}$ и $m_{k}$ так, что

$$
-2^{-k} \leqslant \sum_{j=1}^{n_{k}} \alpha_{j}^{+}+\sum_{j=1}^{m_{k}} \alpha_{j}^{-} \leqslant 2^{-k}
$$

можно осушествить конструкцию, аналогичную изложенной выше и отличающуюся лишш усложнением обозначений. В частности, в правой части (3.6) вместо $e^{-n}$ получим $\exp \left(-\sum_{j=1}^{n_{k}} \alpha_{j}^{+}\right)$, ав (3.7) вместо 1 получим $\exp \left(-\sum_{j=1}^{n_{k}} \alpha_{j}^{+}-\sum_{j=1}^{m_{k}} \alpha_{j}^{-}\right)$. 
Понятно, что указанным образом можно добиться того, чтобы предельными точками $J_{\varepsilon}(\gamma, Q)$ были 0 и $+\infty$ (тогда $J_{\varepsilon}(\gamma, Q)$ будет осциллировать в $\left.(0, \infty)\right)$.

Отметим также, что меру $\gamma$ можно рассматривать не только на счетном произведении прямых, но и на сепарабельном банаховом пространстве $Z$ последовательностей $x=\left(x_{n}\right)$ с нормой $\|x\|=\sup _{n}\left|q_{n} x_{n}\right|$, удовлетворяющих условию $\lim _{n \rightarrow \infty}\left|q_{n} x_{n}\right|=0$. При достаточно быстро убывающей последовательности положительных чисел $q_{n}$ множества $\{q \leqslant \varepsilon\}$ окажутся компактными в $Z$. Из этого явствует, что предел в (0.1) может не существовать для эквивалентных гауссовских мер даже в случае компактных множеств $\{q \leqslant \varepsilon\}$. Объясняется это тем, что согласно сказанному выше (и вопреки сказанному в [5, с. 215]) не всегда соответствующая плотность Радона-Никодима удовлетворяет условию ядерности из теоремы 3.3. Подобные примеры осушествимы на всяком бесконечномерном банаховом пространстве (вообще на всяком локально вьпуклом пространстве $X$, на котором есть гауссовская мера с бесконечномерньм носителем), ибо $Z$ можно вложить в $X$ с помощью непрерывного инъективного линейного оператора. Ситуация, однако, изменится, если на $X$ рассматривать заранее данную норму. Можно также фиксировать и гауссовскую меру, и норму. В этом случае получатся иные классы многочленов второго порядка, для которых существует обсуждаемый предел. Используя [6, предложение 8, с. 287, замечание, с. 288] (см. также [4]), можно заключить, что сушествуют центрированная гауссовская мера $\mu$ на $X=l^{2}$ и последовательность $\left(\alpha_{n}\right) \in l^{2}$ с $\sum_{n=1}^{\infty}\left|\alpha_{n}\right|=\infty$, для которых при $q(x)=\|x\|$ предел в (2.6) положителен и конечен.

Обратимся теперь к случаю, когда $q$ - измеримая полунорма, не обязательно являющаяся нормой на $H$. В этом случае качественно получается такая же картина, лишь значения пределов могут быть иными (это имеет место уже для многочленов первого порядка, см. $[8, \S 4.4])$.

ПРЕДЛОЖЕНИЕ 3.7. Пусть выполнены условия следствия 3.5 за исключением того, что теперь $q-\gamma$-измеримая полунорма, не обязательно являющаяся нормой на $H$. Тогда существует предел $\lim _{\varepsilon \rightarrow 0} J_{\varepsilon}(\gamma, Q)$, причем этот предел положстелен и конечен, если $D_{H}^{2} Q$ - ядерный оператор. В част-

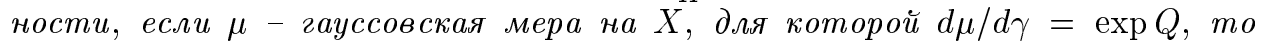
существует предел

$$
\lim _{\varepsilon \rightarrow 0} \frac{\mu(\{q \leqslant \varepsilon\})}{\gamma(\{q \leqslant \varepsilon\})} .
$$

ДокАзАтЕльство. Сохраним обозначения теоремы 3.3. Кроме того, обозначим через $\Gamma$ замкнутое линейное подпространство в $L^{2}(\gamma)$, порожденное последовательностью $\left\{f_{n}\right\}$. Как и выше, достаточно рассмотреть случай, когда $Q=\sum_{n=1}^{\infty} \alpha_{n} \xi_{n}^{2}$, где $\left\{\xi_{n}\right\}$ - ортонормированная последовательность в $X_{\gamma}^{*}, \alpha_{n}>0$ и $\sum_{n=1}^{\infty} \alpha_{n}<\infty$. Тогда

$$
\xi_{n}=\zeta_{n}+\delta_{n}, \quad \zeta_{n} \in \Gamma, \quad \delta_{n} \perp \Gamma
$$

Заметим, что ряды $G_{1}=\sum_{n=1}^{\infty} \alpha_{n} \zeta_{n}^{2}$ и $G_{2}=\sum_{n=1}^{\infty} \alpha_{n} \delta_{n}^{2}$ сходятся почти всюду, ибо $\mathrm{E} \zeta_{n}^{2} \leqslant 1$ и $\mathrm{E} \delta_{n}^{2} \leqslant 1$. Ряд $G_{3}=2 \sum_{n=1}^{\infty} \alpha_{n} \zeta_{n} \delta_{n}$ также сходится почти всюду. Предположим сначала, что $\exp \left(2 G_{1}+2 G_{2}\right) \in L^{1}(\gamma)$. Введем гауссовскую меру

$$
\mu:=\left\|\exp \left(G_{1}+G_{2}\right)\right\|_{L^{1}(\gamma)}^{-1} \exp \left(G_{1}+G_{2}\right) \cdot \gamma
$$


Используя независимость случайных величин $q$ и $G_{2}$ на вероятностном пространстве $(X, \gamma)$, вытекаюшую из ортогональности $\delta_{n}$ всем $f_{j}$ в $L^{2}(\gamma)$, получаем

$$
\begin{aligned}
J_{\varepsilon}(\gamma, Q) & =\frac{\left\|\exp \left(G_{1}+G_{2}\right)\right\|_{L^{1}(\gamma)}}{\gamma\left(V_{\varepsilon}\right)} \int_{V_{\varepsilon}} \exp \left(G_{3}\right) d \mu \\
& =J_{\varepsilon}\left(\mu, G_{3}\right) \frac{\mu\left(V_{\varepsilon}\right)\left\|\exp \left(G_{1}+G_{2}\right)\right\|_{L^{1}(\gamma)}}{\gamma\left(V_{\varepsilon}\right)} \\
& =J_{\varepsilon}\left(\mu, G_{3}\right) J_{\varepsilon}\left(\gamma, G_{1}\right)\left\|\exp \left(G_{2}\right)\right\|_{L^{1}(\gamma)} .
\end{aligned}
$$

Поскольку $G_{1}$ является функцией $\left\{f_{n}\right\}$ и $\sum_{n=1}^{\infty} \alpha_{n}<\infty$, то в силу следствия 3.4 второй сомножитель в правой части вышеприведенного равенства стремится к 1 при $\varepsilon \rightarrow 0$. Покажем, что и первый сомножитель стремится к 1 . Прежде всего заметим, что случайные величины $\zeta_{n}$ и $\delta_{n}$ являются независимыми гауссовскими и относительно меры $\mu$. Это вытекает из их ортогональности, которую легко усмотреть из независимости случайных величин $\zeta_{n} \exp \left(G_{1}\right)$ и $\delta_{n} \exp \left(G_{2}\right)$ на $(X, \gamma)$. Если $\zeta=4 \sum_{j=1}^{k} \alpha_{j} \zeta_{j} \delta_{j}$, то равенство

$$
\lim _{\varepsilon \rightarrow 0} J_{\varepsilon}(\mu, \zeta)=1
$$

вытекает из уже доказанного. Действительно,

$$
\lim _{\varepsilon \rightarrow 0} \frac{1}{\mu\left(V_{\varepsilon}\right)} \int_{V_{\varepsilon}} \zeta^{k} d \mu=0 \quad \forall k \geqslant 1 .
$$

Для доказательства заметим, что ввиду независимости $\delta_{i}$ и $\left\{f_{j}\right\}$ и неравенства Коши-Буняковского проверка этого соотношения сводится к проверке равенства

$$
\lim _{\varepsilon \rightarrow 0} \frac{1}{\mu\left(V_{\varepsilon}\right)} \int_{V_{\varepsilon}} \zeta_{j}^{2 k} d \mu=0 \quad \forall k \geqslant 1
$$

Это равенство следует из того, что $\lim _{\varepsilon \rightarrow 0} J_{\varepsilon}\left(\mu, \zeta_{j}\right)=1$ и функции $\zeta_{j}^{2 k-1}$ имеют нулевые интегралы по множеству $V_{\varepsilon}$ относительно меры $\mu$. Таким образом, величины

$$
s_{k}(\varepsilon):=\frac{1}{k ! \mu\left(V_{\varepsilon}\right)} \int_{V_{\varepsilon}} \zeta^{k} d \mu
$$

стремятся к нулю при каждом $k \geqslant 1$. Кроме того,

$$
\sum_{k=1}^{m}\left|s_{k}(\varepsilon)\right| \leqslant J_{\varepsilon}(\mu, \zeta) \leqslant J_{\varepsilon}\left(\mu, 2 G_{1}+2 G_{2}\right) \leqslant \int_{X} \exp \left(2 G_{1}+2 G_{2}\right) d \mu,
$$

ибо $s_{k}(\varepsilon)=0$ при нечетных $k$, что вытекает из того, что $\zeta_{j}$ и $\delta_{i}$ независимы относительно меры $\mu$ и их нечетные степени имеют равные нулю интегралы (относительно меры $\mu$ ) по множеству $V_{\varepsilon}$. Таким образом, получаем (3.8).

Пусть теперь $q>1$. По теореме Лебега найдется такое $k$, что

$$
\int_{X} \exp \left[\sum_{j=k+1}^{\infty} 2 \alpha_{j}\left(\zeta_{j}^{2}+\delta_{j}^{2}\right)\right] d \mu<q .
$$


Согласно доказанному вьшше найдется такое $\varepsilon_{1}>0$, что для всех $\varepsilon \in\left(0, \varepsilon_{1}\right]$ справедливы неравенства

$$
J_{\varepsilon}\left(\mu, 4 \sum_{j=1}^{k} \alpha_{j} \zeta_{j} \delta_{j}\right)<q, \quad J_{\varepsilon}\left(\mu,-4 \sum_{j=1}^{k} \alpha_{j} \zeta_{j} \delta_{j}\right)<q .
$$

Следовательно,

$$
\begin{aligned}
J_{\varepsilon}\left(\mu, G_{3}\right) & \leqslant \sqrt{J_{\varepsilon}\left(\mu, 4 \sum_{j=1}^{k} \alpha_{j} \zeta_{j} \delta_{j}\right)} \sqrt{J_{\varepsilon}\left(\mu, \sum_{j=k+1}^{\infty} 2 \alpha_{j}\left(\zeta_{j}^{2}+\delta_{j}^{2}\right)\right)} \\
& \leqslant \sqrt{q \int_{X} \exp \left[\sum_{j=k+1}^{\infty} 2 \alpha_{j}\left(\zeta_{j}^{2}+\delta_{j}^{2}\right)\right] d \mu}<q .
\end{aligned}
$$

Аналогично получаем

$$
J_{\varepsilon}\left(\mu,-G_{3}\right) \leqslant q
$$

Поскольку

$$
1=J_{\varepsilon}\left(\mu, \frac{1}{2} G_{3}-\frac{1}{2} G_{3}\right) \leqslant \sqrt{J_{\varepsilon}\left(\mu, G_{3}\right)} \sqrt{J_{\varepsilon}\left(\mu,-G_{3}\right)}
$$

TO

$$
J_{\varepsilon}\left(\mu, G_{3}\right) \geqslant q^{-1},
$$

откуда и вытекает наше утверждение при сделанном вьше дополнительном предположении об интегрируемости $\exp \left(2 G_{1}+2 G_{2}\right)$. В общем случае получаем сушествование предела $J_{\varepsilon}(\gamma, z Q)$ для всех достаточно малых положительных $z$. Кроме того, найдется такое $\lambda>1$, что $\exp (\lambda Q) \in L^{1}(\gamma)$. Тогда в силу леммы 3.1 для всех $z$ из полосы $0 \leqslant \operatorname{Re} z \leqslant \lambda$ функции $f_{\varepsilon}(z)=J_{\varepsilon}(\gamma, z Q), \varepsilon \in(0,1)$, равномерно ограничены. Применяя, как и вьше, теорему Витали, завершаем доказательство.

Изложенное доказательство позволяет получить и явное выражение для предела $J_{\varepsilon}(\gamma, Q)$. Для этого обозначим через $\mathscr{F} \sigma$-алгебру, порожденную последовательностью $\left\{f_{n}\right\}$, задающей полунорму $q$, а через $\mathrm{E}^{\mathscr{F}}$ - условное математическое ожидание относительно $\mathscr{F}$. Кроме того, обозначим через $\Delta \sigma$-алгебру, порожденную ортогональным дополнением к $\left\{f_{n}\right\}$ в $X_{\gamma}^{*}$, а через $\mathrm{E}^{\Delta}-$ соответствуюшее условное математическое ожидание. Отметим, что если $q$ - норма на $H$, то $\mathrm{E}^{\mathscr{F}} f=f$, a $\mathrm{E}^{\Delta}$ совпадает с математическим ожиданием относительно $\gamma$.

СлЕДСТвИЕ 3.8. Пусть выполнены условия предложсения 3.7. Тогда

$$
\lim _{\varepsilon \rightarrow 0} J_{\varepsilon}(\gamma, Q)=\left\|\exp \left(\mathrm{E}^{\Delta} Q\right)\right\|_{L^{1}(\gamma)} \exp \left(-\frac{1}{2} \operatorname{trace} D_{H}^{2} \mathrm{E}^{\mathscr{F}} Q\right)
$$

ДокАЗАТЕЛЬСТво. Если $Q$ не имеет линейного слагаемого, то (3.9) установлено в ходе доказательства предложения 3.7 , ибо $\mathrm{E}^{\mathscr{F}} G_{3}=\mathrm{E}^{\Delta} G_{3}=0$ и

$$
\mathrm{E}^{\Delta} G_{1}=\left(G_{1}, 1\right)_{L^{2}(\gamma)}=\frac{1}{2} \operatorname{trace} D_{H}^{2} G_{1}=\frac{1}{2} \operatorname{trace} D_{H}^{2} \mathrm{E}^{\mathscr{F}} Q
$$


где $G_{2}$ и $G_{3}$ - рассмотренные выше функции. Воспользуемся обозначениями из этого доказательства и запишем $Q$ в виде

$$
\begin{gathered}
Q=c_{0}+\sum_{n=1}^{\infty} c_{n} \xi_{n}+\sum_{n=1}^{\infty} \alpha_{n} \xi_{n}^{2}=Q_{1}+Q_{2} \\
Q_{1}=c_{0}+\sum_{n=1}^{\infty} c_{n} \delta_{n}+\sum_{n=1}^{\infty} \alpha_{n} \delta_{n}^{2}, \quad Q_{2}=\sum_{n=1}^{\infty} c_{n} \zeta_{n}+\sum_{n=1}^{\infty} \alpha_{n} \zeta_{n}^{2}+2 \sum_{n=1}^{\infty} \alpha_{n} \zeta_{n} \delta_{n},
\end{gathered}
$$

где $\left\{\xi_{n}\right\}$ - некоторый ортонормированньй базис в $X_{\gamma}^{*}, \xi_{n}=\zeta_{n}+\delta_{n}, \zeta_{n}$ - ортогональная проекция $\xi_{n}$ на замкнутое линейное подпространство $\Gamma$ в $X_{\gamma}^{*}$, порожденное $\left\{f_{n}\right\}$, причем $\sum_{n=1}^{\infty}\left|\alpha_{n}\right|<\infty$. Кроме того, $Q=f+Q_{0}$, где $f=I_{1}(Q)$ - линейная компонента. Существует такое $\lambda>1$, что $\exp \left(\lambda Q_{0}\right) \in L^{1}(\gamma)$. Пусть $1<\beta<\lambda$. Тогда в силу неравенства Гёльдера, примененного к паре чисел $p=\lambda / \beta$ и $q=p(p-1)^{-1}$, имеем

$$
J_{\varepsilon}(\gamma, \beta Q) \leqslant J_{\varepsilon}\left(\gamma, \lambda Q_{0}\right)^{1 / p} J_{\varepsilon}(\gamma, q f)^{1 / q}
$$

Из леммы 3.1 вытекает равномерная ограниченность $J_{\varepsilon}\left(\gamma, \lambda Q_{0}\right)$ по $\varepsilon \in(0,1]$, а [8, лемма 4.4.1] дает равномерную ограниченность $J_{\varepsilon}(\gamma, q f)$. Следовательно, голоморфные функции $f_{\varepsilon}(z)=J_{\varepsilon}(\gamma, Q), \varepsilon \in(0,1]$, равномерно ограничены в области $0<\operatorname{Re} z<\beta$. В силу теоремы Витали достаточно показать, что при малых положительных $z$ и $\varepsilon \rightarrow 0$ функции $f_{\varepsilon}(z)$ стремятся к голоморфной функции

$$
\left\|\exp \left(z \mathrm{E}^{\Delta} Q\right)\right\|_{L^{1}(\gamma)} \exp \left(-\frac{z}{2} \operatorname{trace} D_{H}^{2} \mathrm{E}^{\mathscr{F}} Q\right)
$$

Далее считаем, что $z \in[0, r]$, где $r \in(0,1 / 2)$ таково, что $\exp \left(2 r Q_{1}\right) \in L^{1}(\gamma)$ и $\exp \left(2 r Q_{2}\right) \in L^{1}(\gamma)$. Отметим, что

$$
\lim _{\varepsilon \rightarrow 0} J_{\varepsilon}\left(\gamma, z Q_{2}\right)=1 \quad \forall z \in[0,2 r] .
$$

Действительно, $z Q_{2}=l+G$, где

$$
l=z \sum_{n=1}^{\infty} c_{n} \zeta_{n}, \quad G=z \sum_{n=1}^{\infty} \alpha_{n} \zeta_{n}^{2}+2 z \sum_{n=1}^{\infty} \alpha_{n} \zeta_{n} \delta_{n} .
$$

Введем центрированную гауссовскую меру $\nu:=\|\exp G\|_{L^{1}(\gamma)}^{-1} \exp G \cdot \gamma$. Тогда $J_{\varepsilon}\left(\gamma, z Q_{2}\right)=J_{\varepsilon}(\nu, l) J_{\varepsilon}(\gamma, G)$. Первый сомножитель в правой части этого равенства стремится к 1 при $\varepsilon \rightarrow 0$ согласно [8, теорема 4.4.3], поскольку $l$ принадлежит замкнутому линейному подпространству, порожденному функционалами, задающими полунорму $q$. Второй сомножитель стремится к 1 на основании доказанного вьше. Введем теперь вероятностную меру

$$
\gamma_{\varepsilon}:=\gamma\left(V_{\varepsilon}\right)^{-1} I_{V_{\varepsilon}} \cdot \gamma
$$

Проинтегрируем по этой мере тождество

$$
\exp (z Q)=\exp \left(z Q_{1}\right)+\exp \left(z Q_{1}\right)\left(\exp \left(z Q_{2}\right)-1\right)
$$


Ввиду независимости случайных величин $Q_{1}$ и $q$, вытекающей из ортогональности $\left\{\delta_{i}\right\}$ и $\left\{f_{j}\right\}$, интеграл от функции $\exp \left(z Q_{1}\right)$ совпадает с ее интегралом по мере $\gamma$. Интеграл от второго слагаемого в правой части (3.11) оценивается следующим образом с помощью неравенства Коши-Буняковского:

$$
\begin{aligned}
& \left|\int_{X} \exp \left(z Q_{1}\right)\left(\exp \left(z Q_{2}\right)-1\right) d \gamma_{\varepsilon}\right| \\
& \quad \leqslant \sqrt{J_{\varepsilon}\left(\gamma, 2 z Q_{1}\right)} \sqrt{J_{\varepsilon}\left(\gamma, 2 z Q_{2}\right)-2 J_{\varepsilon}\left(\gamma, z Q_{2}\right)+1} \\
& \quad=\sqrt{\left\|\exp \left(2 z Q_{1}\right)\right\|_{L^{1}(\gamma)}} \sqrt{J_{\varepsilon}\left(\gamma, 2 z Q_{2}\right)-2 J_{\varepsilon}\left(\gamma, z Q_{2}\right)+1}
\end{aligned}
$$

Ввиду (3.10) правая часть этой оценки стремится к нулю при $\varepsilon \rightarrow 0$. Осталось заметить, что интеграл $\exp \left(z Q_{1}\right)$ по мере $\gamma$ совпадает с правой частью $(3.9)$ с функцией $z Q$ вместо $Q$. Действительно, обозначая через $\mathrm{E}$ математическое ожидание относительно $\gamma$ и пользуясь независимостью $\left\{\delta_{n}\right\}$ и $\left\{\zeta_{n}\right\}$, получаем

$$
\mathrm{E}^{\Delta} Q=c_{0}+\sum_{n=1}^{\infty} \alpha_{n} \delta_{n}+\sum_{n=1}^{\infty} \alpha_{n} \delta_{n}^{2}+\sum_{n=1}^{\infty} \alpha_{n} \mathrm{E} \zeta_{n}^{2} .
$$

С другой стороны, как отмечалось в доказательстве следствия 3.4 , при наших условиях справедливо равенство (2.4) для функции $\sum_{n=1}^{\infty} \alpha_{n} \zeta_{n}^{2}$, что дает

$$
\sum_{n=1}^{\infty} \alpha_{n} \mathrm{E} \zeta_{n}^{2}=\frac{1}{2} \operatorname{trace} D_{H}^{2}\left(\sum_{n=1}^{\infty} \alpha_{n} \zeta_{n}^{2}\right)=\frac{1}{2} \operatorname{trace} D_{H}^{2} \mathrm{E}^{\mathscr{F}} Q \text {. }
$$

Отметим еще, что в (3.9) можно не привлекать $\mathrm{E}^{\mathscr{F}}$, поскольку

$$
\operatorname{trace} D_{H}^{2} \mathrm{E}^{\mathscr{F}} Q=\operatorname{trace} D_{H}^{2} Q-\operatorname{trace} D_{H}^{2} \mathrm{E}^{\Delta} Q \text {. }
$$

Как показано в [3], для всякой строго положительной абсолютно непрерывной функции $\varphi$, имеющей производную ограниченной вариации на $[0,1]$ и удовлетворяющей условию

$$
\int_{0}^{1} \varphi(t)^{-2} d t=1
$$

существует конечный положительный предел

$$
\lim _{\varepsilon \rightarrow 0} \frac{P^{W}(x:|x(t)| \leqslant \varepsilon \varphi(t) \forall t \in[0,1])}{P^{W}\left(x: \sup _{t \in[0,1]}|x(t)| \leqslant \varepsilon\right)} .
$$

Этот результат можно вывести из доказанного выше. Для этого заметим, что числитель в левой части (3.12) представим в виде $P^{W}(x: q(T x) \leqslant \varepsilon)$, где

$$
T x(t)=\frac{1}{\sqrt{\tau^{\prime}(t)}} x(\tau(t))
$$

причем $\tau$ - функция, обратная к

$$
\beta(t)=\int_{0}^{t} \varphi(s)^{-2} d s
$$


Действительно, неравенство $|x(t)| \leqslant \varepsilon \varphi(t) \forall t \in[0,1]$ равносильно неравенству $|x(\tau(t))| \leqslant \varepsilon \varphi(\tau(t)) \forall t \in[0,1]$, а значит, неравенству $|x(\tau(t))| / \sqrt{\tau^{\prime}(t)} \leqslant \varepsilon$ $\forall t \in[0,1]$, ибо $\varphi(\tau(t))=\sqrt{\tau^{\prime}(t)}$ в силу правила дифференцирования обратной функции и (3.13). Как известно [18], мера $P^{W} \circ T^{-1}$ эквивалентна мере $P^{W}$. Этот факт вытекает из того, что оператор $T$ на пространстве Камерона-Мартина $H$ меры $P^{W}$ имеет вид $T=U(I+K)$, где $U$ - ортогональный оператор в $H$, а $K-$ оператор Гильберта-Шмидта в $H$. Напомним, что $H$ состоит из абсолютно непрерывных функций $h$, удовлетворяющих условиям $h(0)=0$ и $h^{\prime} \in L^{2}[0,1]$. При этом $|h|_{H}=\left\|h^{\prime}\right\|_{L^{2}[0,1]}$. Как несложно убедиться, условие применимости теоремы 3.3 состоит в ядерности квадратичной формы $(T h, T h)_{H}-(h, h)_{H}$ на $H$. Непосредственные вычисления дают следующее представление:

где

$$
(T h, T h)_{H}-(h, h)_{H}=\int_{0}^{1} \psi(t) h(t) h^{\prime}(t) d t+\int_{0}^{1} \theta(t) h(t) h(t) d t,
$$

$$
\psi(t)=-\frac{\tau^{\prime \prime}(\beta(t))}{\tau^{\prime}(\beta(t))^{2}}, \quad \theta(t)=\frac{1}{4} \frac{\tau^{\prime \prime}(\beta(t))^{2}}{\tau^{\prime}(\beta(t))^{4}} .
$$

Второй интеграл в правой части задает квадратичную форму, порождаемую некоторьм ядерным оператором, поскольку эта форма является ограничением непрерывной формы на $C[0,1]$ (см. лемму 2.5). Заметим, что $\tau^{\prime}(\beta(t)) \beta^{\prime}(t)=1$ и $\tau^{\prime \prime}(\beta(t)) \beta^{\prime}(t)^{2}+\tau^{\prime}(\beta(t)) \beta^{\prime \prime}(t)=0$, откуда $\tau^{\prime \prime}(\beta(t))=2 \varphi^{\prime}(t) \varphi(t)^{3}$. Если $\varphi^{\prime}$ имеет ограниченную вариацию, то такова же и $\psi$. Значит, первый интеграл является ограничением непрерывной квадратичной формы на $C[0,1]$, что означает, что эта форма задается ядерным оператором на $H$. Следует отметить, однако, что в работе [2] анонсировано выражение для предела в (3.12) при более слабом условии квадратичной интегрируемости $\varphi^{\prime}$.

ЗАмечАние 3.9. а) В работах [17], [19] для оценивания условных экспоненциальных моментов функций разных классов было предложено использовать так называемые неравенства корреляции типа

$$
\gamma(A \cap B) \geqslant \gamma(A) \gamma(B),
$$

известные для некоторых пар выпуклых симметричных множеств $A$ и $B$ (например, в качестве $A$ можно взять любое выпуклое симметричное множество, а в качестве $B$ - симметричную полосу вида $|f| \leqslant c$, где $f$ - линейная функция; см. [20]). Эти неравенства использовались и в [5].

б) В бесконечномерном случае может не существовать нетривиального предела

$$
\gamma(\varepsilon U)^{-1} \int_{\varepsilon U} f d \gamma
$$

даже для единичного шара $U$ в гильбертовом пространстве с гауссовской мерой $\gamma$ и функции $f \in L^{1}(\gamma)$. Например, в [21] построены такие центрированная гауссовская мера $\gamma$ на сепарабельном гильбертовом пространстве $X$ и множество $A$ положительной $\gamma$-меры, что

$$
\lim _{\varepsilon \rightarrow 0} \frac{\gamma(A \cap B(x, \varepsilon))}{\gamma(B(x, \varepsilon))}=0 \quad \gamma \text {-п.в., }
$$

где $B(x, \varepsilon)$ - замкнутый шар радиуса $\varepsilon$ с центром в $x$. В работах [21]-[23] можно найти и другие контрпримеры на эту тему. 


\section{Список литературы}

1. Сытая Г. Н. Об асимптотике меры малых сфер для гауссовских процессов, эквивалентных броуновскому // Теория вероятн. и матем. статист. 1977. № 19. С. 128-133.

2. Нагаев C. B. Об асимптотике винеровской меры узкой полосы // Теория вероятн. и ее примен. 1981. Т. 26. №3. С. 630.

3. Новиков A. А. Малые уклонения гауссовских процессов // Матем. заметки. 1981. Т. 29. № 2. C. 291-301.

4. $L i W$. Comparison results for the lower tail of Gaussian seminorm // J. Theoret. Probab. 1992. V. 5. P. 1-32.

5. Лифиич М. А. Гауссовские случайные функции. Киев: ТВиМС, 1995.

6. Linde $W$. Comparison results for the small ball behavior of Gaussian random variables // Probability in Banach spaces / ed. J. Hoffmann-Jorgensen, J. Kuelbs, M. B. Marcus. V. 9. Boston: Birkhäuser, 1994. P. 273-297.

7. Богачев В.И. Функции Онзагера-Маклупа для гауссовских мер // Докл. АН. 1995. Т. 344. № 4. C. 439-441.

8. Богачев В. И. Гауссовские меры. М.: Физматлит, 1997.

9. Kuelbs J., Li W. Metric entropy and the small ball problem for Gaussian measures // J. Funct. Anal. 1993. V. 116. № 1. P. 133-157.

10. Shao Q.-M., Wang D. Small ball probabilities of Gaussian fields // Probab. Theory Related Fields. 1995. V. 102. P. 511-517.

11. Shi Z . Small ball probabilities for a Wiener process under weighted sup-norms, with an application to the supremum of Bessel local times // J. Theoret. Probab. 1996. V. 9. № 4 . P. 915-929.

12. Stolz $W$. Some small ball probabilities for Gaussian processes under nonuniform norms // J. Theoret. Probab. 1996. V. 9. №3. P. 613-630.

13. Cirelson B. S., Ibragimov I. A., Sudakov V. N. Norms of Gaussian sample functions // Lect. Notes in Math. 1976. V. 550. P. 20-41.

14. Ширяев А. Н. Вероятность. М.: Наука, 1989.

15. Вахания Н.Н., Тариеладзе В.И., Чобанян С.А. Вероятностные распределения в банаховых пространствах. М.: Наука, 1984.

16. Титчмари Е. Теория функций. М.: Наука, 1980.

17. Shepp L.A., Zeitouni O. A note on conditional exponential moments and the Onsager Machlup functional // Ann. Probab. 1992. V. 20. № 1. P. 652-654.

18. Shepp L. A. Radon-Nikodym derivatives of Gaussian measures // Ann. Math. Statist. 1966. V. 37. № 2. P. 321-354.

19. Shepp L. A., Zeitouni O. Exponential estimates for convex norms and some applications // Barcelona Seminar on Stochastic Analysis. Progr. Probab. V. 32. Boston: Birkhäuser, 1993. P. 203-215.

20. Sidák $Z$. On multivariate normal probabilities of rectangles: their dependence on correlations // Ann. Math. Statist. 1968. V. 39. № 5. P. 1425-1434.

21. Preiss D. Gaussian measures and covering theorems // Comment. Math. Univ. Carolin. 1979. V. 20. № 1. P. 95-99.

22. Preiss D. Gaussian measures and the density theorems // Comment. Math. Univ. Carolin. 1981. V. 22. № 1. P. 181-193.

23. Preiss D. Differentiation of measures in infinitely dimensional spaces // Proc. Conf.: Topol. and Meas. III, Vitte/Hiddensee, Oct. 19-25, 1980. Part 2, Greifswald. Wissen. Beitrage d. Greifswald Univ., 1982. P. 201-207.

Московский государственный

Поступила в редакцию

университет им. М.В. Ломоносова

05.02 .1998 